\section{Hsp90 Inhibition Affects Cell Metabolism by Disrupting Mitochondrial Protein Insertion}

\section{Maria E Mycielska ${ }^{1 *}$, Christian J Wachsmuth ${ }^{2}$, Katja Dettmer ${ }^{2}$, Christine Wagner ${ }^{1}$, Hans J Schlitt ${ }^{1}$, Peter J Oefner ${ }^{2}$, Edward K Geissler ${ }^{1}$ and Sven A Lang ${ }^{1}$}

${ }^{1}$ Department of Surgery, University Hospital Regensburg, University of Regensburg, Regensburg, Germany

2Institute of Functional Genomics, University of Regensburg, Regensburg, Germany

\begin{abstract}
Hsp90 is a molecular chaperone interacting with hundreds client proteins. Little is known, however, about Hsp90 influence on cancer cell metabolism. Here we show that the inhibition of Hsp90 affects indirectly glycolysis by disrupting mitochondrial insertion of the elements of Translocase of the Outer Membrane (TOM) complex in tumor cells. Improper insertion of Tom40 decreases the abundance of many mitochondrial proteins resulting in reduced mitochondrial activity. This is accompanied by increased production and release of lactate and serine. These results indicate an increased rate of glycolysis with serine synthesis compensating for the loss of energy and mitochondrially derived metabolites, potentially enhancing the metastatic potential of surviving cells. Our results provide novel insights into the effects of Hsp90 inhibition on cancer cell metabolism.
\end{abstract}

Keywords: Cancer; Glycolysis; Mitochondrial activity; Tom40

\section{Introduction}

Molecular chaperones form complexes with client proteins to assure proper folding and trafficking [1]. Heat-shock protein 90 (Hsp90) is a conserved chaperone that interacts with hundreds of clients. Cancer cells require Hsp90 to protect and keep oncoproteins in appropriate forms necessary for their activity and survival [2].

Cancer cells are known to have an altered metabolism, with glycolysis becoming their predominant means of acquiring energy

*Corresponding author: Maria E Mycielska, Department of Surgery, University Hospital Regensburg, Franz-Josef-Strauss Allee 11, 93053 Regensburg, Germany, Tel: +499419444879; Fax: +499419444877; E-mail: maria.mycielska@ukr.de

Citation: Mycielska ME, Wachsmuth CJ, Dettmer K, Wagner C, Schlitt HJ, et al. (2014) Hsp90 Inhibition Affects Cell Metabolism by Disrupting Mitochondrial Protein Insertion. J Cell Biol Cell Metab 1: 002.

Received: July 08, 2014; Accepted: July 31, 2014; Published: August 14, 2014 even in the presence of oxygen [3]. This switch in energy production is called the "Warburg effect" [4]. In cancer cells, one of the most important enzymes in the process of glycolysis is Hexokinase II (HK II). In particular, a strong correlation between HK II expression and tumor size, differentiation, as well as tumor stage, has been shown in gastric cancer [5]. Furthermore, the malignant potential of pancreatic cancer has been correlated to the expression of HK II in the tumor tissue [6]. While HK II is responsible for metabolizing glucose into glucose-6-phosphate [7], the enzyme activity depends on its subcellular localization. HK II has greater activity when attached to the Outer Mitochondrial Membrane (OMM) through Voltage Dependent Anion Channels (VDACs); this localization facilitates access of the enzyme to the ATP pool [8]. Interestingly, facilitated detachment of HK II from VDAC in cancer cells was found to be necessary in increasing mitochondrial activity and therefore, shifting cellular metabolism towards oxidative phosphorylation [9].

The main question of the present study was to determine the effects of blocking Hsp90 on cancer cell metabolism. Hsp90 is also present in non-transformed cells; however, its activity in non-cancerous cells was not the subject of the present study. Some reports have shown that Hsp90 does not affect glucose uptake, which would exclude a direct interaction of Hsp90 with plasma membrane glucose transporters [10]. Also, proteomic studies of the cells treated with Hsp90 inhibitors did not reveal any changes in HK II expression [11]. Hsp90 affects the assembly of elements of the mitochondrial TOM complex into mitochondrial membranes [12,13]. The latter underlies the hypothesis of the present study that disruption of the TOM complex by Hsp90 inhibition will result in a lower abundance of functional VDACs in the mitochondrial membrane and, as a consequence, in reduced glycolytic activity of the cells. Here we provide a mechanism by which blocking Hsp90 affects Tom 40 , VDAC and HK II abundance in mitochondria, as well as the influence of this mitochondrial imbalance on cancer cell metabolism.

\section{Materials and Methods}

\section{Cell culture}

MiaPaCa-2 pancreatic cancer cells and TMK-1 gastric cancer cells were obtained from ATCC (Manassas, VA, USA). The murine pancreatic cancer cell line Panc02 was kindly provided by Prof. Volker Schmitz (University of Bonn, Bonn, Germany). Cells were grown in DMEM medium with $15 \%$ (MiaPaCa-2) and 10\% (TMK-1 and Panc02) fetal bovine serum supplemented with glutamine, pen-strep and vitamins. Cells were grown in $10 \mathrm{~cm}$ petri dishes (unless otherwise indicated in the text) and maintained in a humidified incubator kept at $37^{\circ} \mathrm{C}$ with $5 \% \mathrm{CO}_{2}$. The water-soluble Hsp90 inhibitor 17-DMAG (17-Dimethylaminoethylamino-17demethoxygeldanamycin) was obtained from Invivogen (Toulouse, France).

\section{Transient siRNA transfection}

To inhibit Tom40 expression, transient down-regulation by

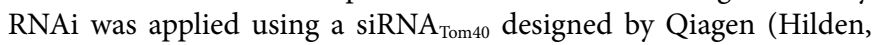
Germany). TMK-1 and MiaPaCa-2 cancer cells were plated at about $50 \%$ cell density and were transiently transfected with siRNA $_{\text {Tom } 40}$ 
using Lipofectamine (Qiagen), according to the manufacturer's instructions. Administration of Lipofectamine (mock) or scrambled RNA (neg. ctrl.) served as controls. Knock-down was confirmed by Western blotting for Tom 40 .

\section{Extraction of mitochondrial protein and determination of hexokinase activity}

Mitochondria were extracted in two different ways. a) For protein expression studies, cells were grown in $75-\mathrm{cm}^{2}$ flasks and incubated for $72 \mathrm{~h}$ before cell harvest in the presence or absence of 17-DMAG (100 nM). A mitochondria isolation kit (Qiagen) was used to protein. b) The hexokinase-mitochondrial fraction was extracted as described by [14]. Enzyme activity was measured in cytosolic and mitochondrial fractions separately using a VarioScan spectrophotometer from Thermo Scientific (Waltham, MA, USA). The measurements were recorded at $30^{\circ} \mathrm{C}$, every 10 seconds for one hour. Enzyme activity was assessed as described by [14]

\section{Western blotting and co-immunoprecipitation}

Western blotting was performed from cytosolic and mitochondrial protein as described before $[15,16]$. Antibodies to HK I, HK II, HK III, Hsp90, Tom40, VDAC (for all 3 isoforms), PDI and $\beta$-actin were purchased from Santa Cruz Biotechnology (Dallas, TX, USA).

For co-Immunoprecipitation (co-IP) experiments, cells were left untreated (control) or cultured in the presence of 17-DMAG (100 $\mathrm{nM})$. Briefly, $500 \mu \mathrm{g}$ of mitochondrial protein together with $1 \mu \mathrm{g}$ of Tom40-specific antibody was mixed for $1 \mathrm{~h}$ at $4^{\circ} \mathrm{C}$. After incubation, $25 \mu \mathrm{L}$ of agarose beads (Santa Cruz Biotechnology) were added and samples were mixed overnight at $4^{\circ} \mathrm{C}$ in elution buffer from Pierce Co-Immunoprecipitation Kit (Thermo Scientific, Waltham, MA, USA). Samples were then washed 5 times with $500 \mu \mathrm{L}$ elution buffer and protein was collected; Western blotting was performed as described previously $[15,16]$

\section{Immunocytochemistry}

Cell staining was performed as described before [17]. Briefly, cells were grown in 24-well plates on glass inserts. For all types of staining, cells were fixed in $5 \%$ paraformaldehyde, permeabilised with $0.1 \%$ saponin, blocked with antibody dilution buffer (DCS Innovative Diagnostik-Systeme, Hamburg, Germany) and incubated with Mitotracker (Invitrogen, Carlsbad, CA, USA) for 30 minutes followed by application of the appropriate antibody specific for VDAC, HK II or Tom40 (Santa Cruz Biotechnologies) for 1 hour. Coverslips were then mounted in DAKO (Glostrup, Denmark) fluorescent-mounting media. Photos were taken using a Zeiss Axio Vision microscope (Oberkochen, Germany) and the analysis performed using ImageJ software. For each condition at least 150 cells were observed from 3 independent experiments.

\section{Metabolomics}

For metabolic analysis, 500,000 cells were plated into $10 \mathrm{~cm}$ petri dishes and treated with 100nM 17-DMAG, siRNA ${ }_{\text {Tom 40 }}$, control siRNA (Qiagen), and control medium, respectively. After $24 \mathrm{~h}$, cells were washed once with PBS and media containing $4 \mathrm{mM}\left[\mathrm{U}_{-}{ }^{13} \mathrm{C}\right]$ glucose was added. After $48 \mathrm{~h}$, cells were harvested and extracted as previously described [18] using $10 \mu \mathrm{L}$ of $1 \mathrm{mM} \quad\left[{ }^{2} \mathrm{H}_{7}\right]$ trans-cinnamic acid (Sigma-Aldrich/Fluka, Taufkirchen, Germany) as extraction standard. Samples were reconstituted in $200 \mu \mathrm{L}$ of water for HPLC-MS/MS analysis, and $10 \mu \mathrm{L}$ of this extract was injected into an Agilent 1200 SL HPLC system (Böblingen, Germany) coupled to a ABSciex API 4000 QTrap mass spectrometer (ABSciex, Darmstadt, Germany) equipped with a TurboV electrospray ion source operated in negative mode. A Phenomenex Luna NH2 $(150 \times 2 \mathrm{~mm}$ i.d., $3 \mu \mathrm{m}$, Torrence, CA, USA) column with mobile phases A $[0.1 \%(\mathrm{v} / \mathrm{v})$ formic acid (Sigma-Aldrich) in PURELAB Plus water] and B [acetonitrile (LC-MS grade, Sigma-Aldrich)] at $250 \mu \mathrm{L} / \mathrm{min}$ was used employing the following gradient: $100 \% \mathrm{~A}(8 \mathrm{~min}) ; 100 \% \mathrm{~A}$ to $0 \% \mathrm{~A}$ from $8-10$ $\mathrm{min} ; 0 \% \mathrm{~A}(5 \mathrm{~min}) ; 0 \%$ A to $100 \%$ A from $15-16 \mathrm{~min} ; 100 \% \mathrm{~A}$ (6 min). MS/MS analysis was performed in Multiple Reactions Monitoring (MRM) using one transition each for the unlabelled analyte and the labelled analogue(s). Ion ratios were calculated from the sum of the peak areas of monitored labelled substrates divided by the area of the unlabeled analogue. For succinate +2 and +4 isotopes were combined throughout all labelling experiments. For overall statistical analyses, two-sided t-tests were used assuming unequal variances between groups.

Labelled and unlabelled serine and glycine in $10 \mu \mathrm{L}$ of media were derivatized with propyl chloroformate and analysed by HPLC-ESI-MS/MS as described previously [19]. The MRM transitions were programmed separately for unlabeled and fully labelled serine and glycine. To determine lactate release, $10 \mu \mathrm{L}$ of medium were dried by means of a vacuum evaporator (CombiDancer, Hettich AG, Bäch, Switzerland), subjected to methoximation and silylation and analyzed by GC EI-qMS [20]. Lactate ion ratios were calculated from ions $\mathrm{m} / \mathrm{z} 219$ and 222 for unlabeled and fully labelled lactate derivative, respectively.

\section{Animal experiments}

For in vivo experiments, $1 \times 10^{5}$ murine Pancreatic cancer cells (Panc02) were injected into the right flank of BL6 mice $[15,16]$. Treatment was initiated after 8 days when tumors had reached a size of $90-100 \mathrm{~mm}^{3}$. Mice received an initial loading dose of $25 \mathrm{mg} / \mathrm{kg}$ $17-D M A G$ and, thereafter, a maintenance dose of $10 \mathrm{mg} / \mathrm{kg} 17-\mathrm{DMAG}$ every other day via intraperitoneal injection. Mice were sacrificed after 17 days of treatment and tumors were excised for analysis.

\section{Statistics}

The data are presented as the average $\pm S D ; n \geq 3$. To assess statistical significance treated samples were compared to control values. For the experiments in (Figures 1-3) ANOVA followed by a TukeyHSD test was used for multiple group comparison. For all the other experiments unpaired $t$-test was employed.

\section{Results}

\section{Hexokinase activity in cancer cell lines}

Measurements of the enzymatic activity of hexokinase comprised the following types of hexokinases: HK I, II and III. Total hexokinase activity was measured in the cytosolic and mitochondrial fractions. In both human cancer cell lines (TMK-1, MiaPaCa-2), no difference in hexokinase activity in the cytosolic fractions was observed upon treatment with the Hsp90 inhibitor 17-DMAG (100 nM) (data not shown). In contrast, there was a significant decrease in hexokinase activity in the mitochondrial fractions of both human cancer cell lines treated with 17-DMAG compared to their untreated controls (Figure 1a and 1b). In parallel, hexokinase activity in TMK-1 and MiaPaCa-2 cancer cells transiently transfected with siRNA against Tom40 was determined. Similar to the treatment with 17-DMAG, a significant decrease in hexokinase activity in the mitochondrial fractions was observed (Figure 1a and 1b). Again, no hexokinase 
activity difference in the cytosolic fractions was found when compared to control samples (data not shown). To exclude the possibility that these two treatments were acting through different pathways, 17-DMAG was applied to cells together with siRNA against Tom40. Results show that this possibility is unlikely, as the combination of Hsp90 inhibition with Tom40 knock-down did not increase the effect compared to either treatment applied alone (Figure 1a and $1 \mathrm{~b}$ ).

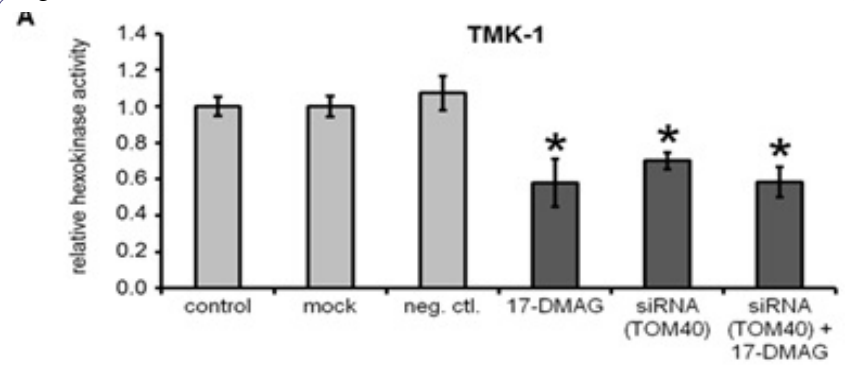

B

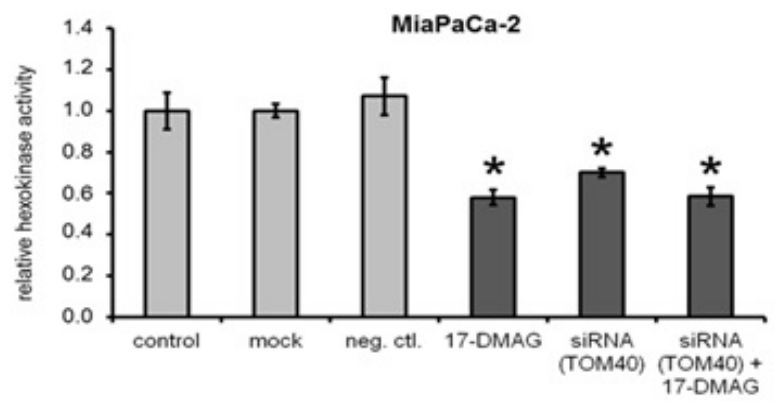

Figure 1: Relative changes in total hexokinase activity in the mitochondrial fraction.

Total hexokinase activity measured in the mitochondrial fractions of a) TMK-1 and b) MiaPaCa-2 cells was significantly reduced upon treatment with Hsp90 inhibitor 17-DMAG (100nM), Tom40 blockade or both treatments together. Enzyme activity was measured after $72 \mathrm{~h}$. Regular media conditions, transfection reagent (mock) and scrambled siRNA (neg. ctl.) served as controls. Differences in the total hexokinase activity between different conditions are shown as relative changes compared to normalized control ( $n=3$ for each experiment; ${ }^{*} P<0.05$ versus control).

\section{Subcellular localisation of Tom40, VDAC and HK II}

Tom40: Tom40 is an integral part of TOM machinery known to localize to mitochondria and has been shown to be an Hsp90 client protein. The aim of the following experiments was to determine in both human cancer cell lines potential changes in localization of Tom40, compared to control conditions, upon treatment with 17-DMAG or siRNA-mediated Tom40 knockdown. Under control conditions, Tom40 largely co-localized with mitochondria (Figure 2a and $2 \mathrm{~b}$ top row). In contrast, cells treated with 17-DMAG showed a more dispersed distribution of Tom 40 protein compared to control conditions (Figure $2 \mathrm{a}$ and $2 \mathrm{~b}$ middle row). This observation is also reflected by a significant increase in Tom40 area upon Hsp90 blockade (Figure 2a and 2b right side). Finally, direct blockade of Tom 40 with siRNA led to a visible reduction in Tom40 staining intensity and only a minor change in the area of staining, as trafficking of the protein was not affected (Figure 2a and 2b). Together, these results suggest that $\mathrm{Hsp} 90$ inhibition causes changes in localization of Tom 40 by preventing its successful trafficking/ assembly in the mitochondrial membrane.

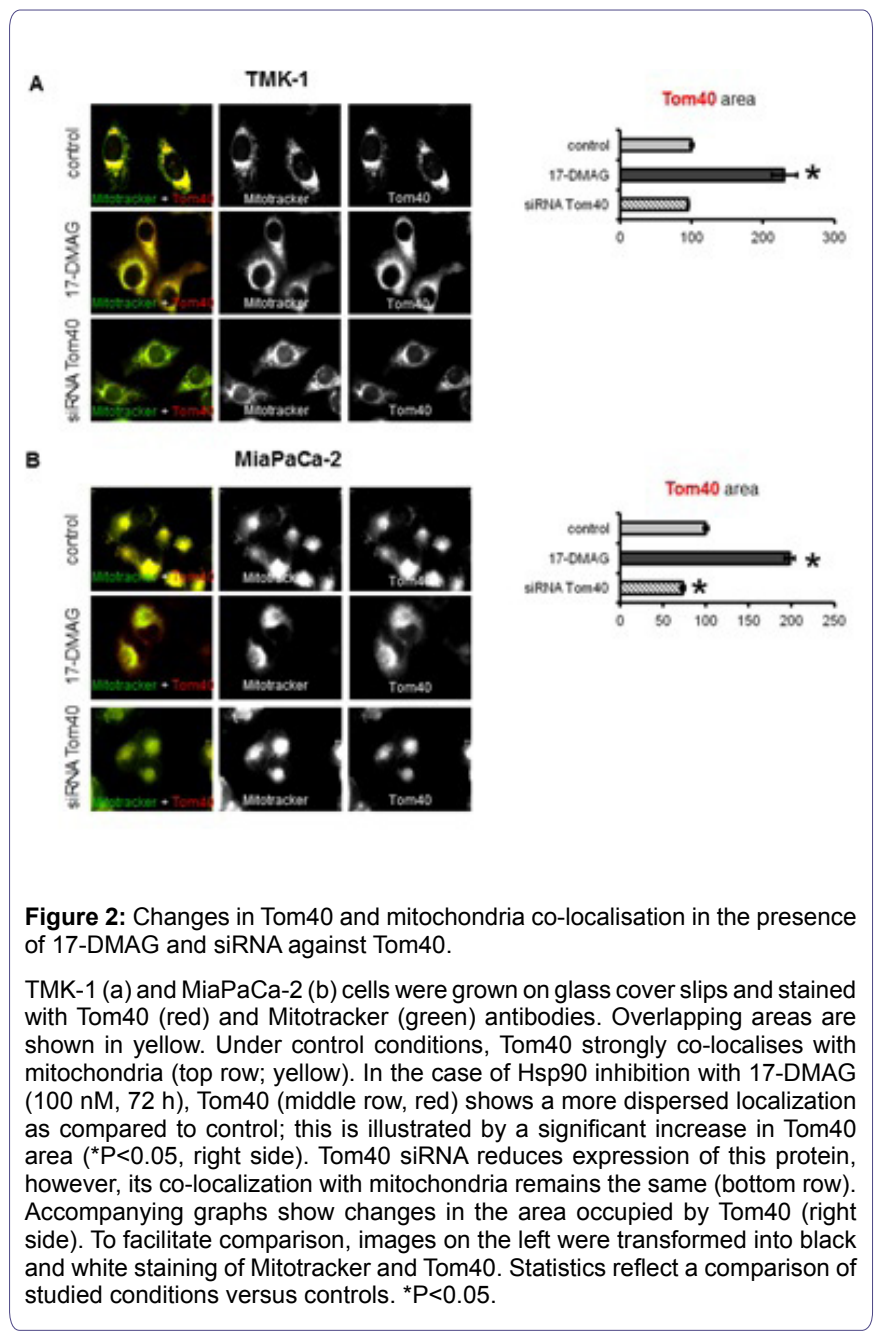

HK II and VDAC: TOM complex facilitates membrane transfer of mitochondrial proteins coded in the nucleus including VDACs. VDACs on the other hand bind HK II and supply ATP necessary for its activity. While our previous results describe the changes in the localization and expression of Tom 40 upon Hsp90 inhibition, it was necessary to assess the consequences of these changes on subcellular localisation of VDACs and HK II. Under control conditions HK II and VDACs co-localised to mitochondria and to each other (Figure 3a, and $3 \mathrm{~b}$ top row). Co-localization of VDACs with HK II was confirmed by the fact that the areas occupied by these two proteins were essentially the same (within a 5\% difference) for TMK-1 and MiaPaCa-2 cells (Figure $3 a$ and $3 b$ ). Upon treatment with 17-DMAG for $72 \mathrm{~h}$, co-localization of VDACs with HK II was reduced (Figure 3a and $3 \mathrm{~b}$ middle row). Accordingly, the area covered by the HK II and VDAC staining was significantly increased. This result was consistent with the changes in subcellular localisation of HK II and VDACs, and a more dispersed localisation of these proteins (less overlap with mitochondria). Interestingly, a similar effect was observed when the cells were transiently transfected with Tom 40 siRNA (Figure 3a and $3 \mathrm{~b}$ bottom row). Again, the area of staining for VDACs and HK II increased, whilst co-localization of these proteins with mitochondria was decreased. However, compared to 17-DMAG treatment, this increase was somewhat less pronounced. In conclusion, Hsp90 inhibition affects subcellular distribution of VDACs and HK II in cancer cells via a Tom40-dependent mechanism.

Volume $1 \cdot 100002$ 
A

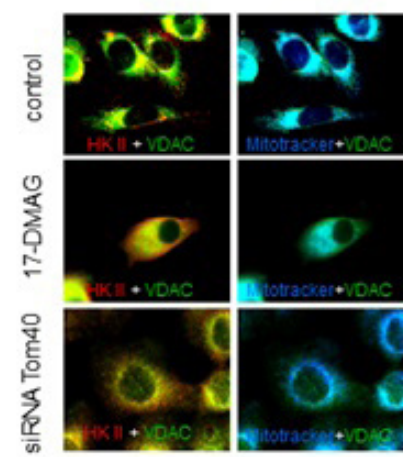

B

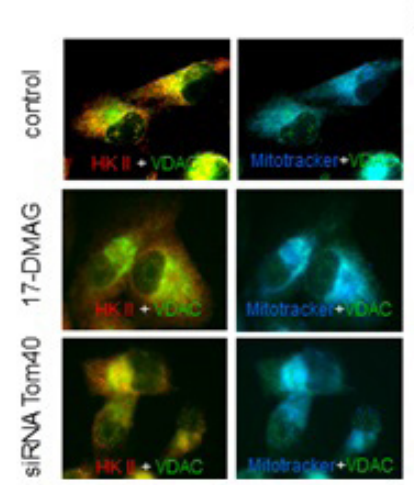

TMK-1

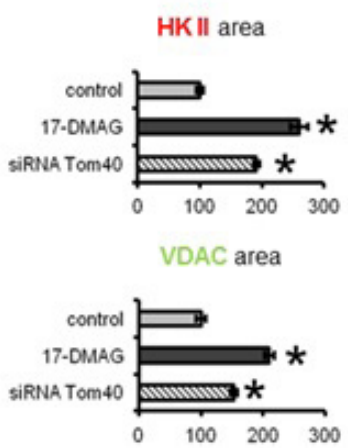

MiaPaCa-2
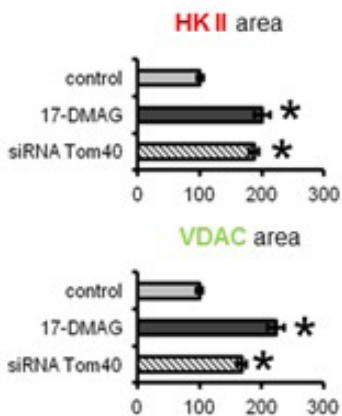

Figure 3: Changes in VDAC co-localisation with HK II in the presence of 17-DMAG and siRNA against Tom40.

TMK-1 (a) and MiaPaCa-2 (b) cells were stained with HK II (red), VDAC (green) and Mitotracker (blue). Under control conditions, VDACs co-localize with mitochondria and HK II (top row). This changes to a more dispersed localization upon treatment with Hsp90 inhibitor and Tom40 knockdown (middle and bottom row), as indicated by a significant increase in HK II and VDAC area ( ${ }^{*} \mathrm{P}<0.05$ versus control, right side). Accompanying graphs show changes in the area occupied by VDACs and HK II (right side). Statistics reflect a comparison of studied conditions versus controls. ( $\left.{ }^{*} P<0.05\right)$.

\section{Expression level of Tom40, HKs, VDACs and Hsp90}

To further assess these issues, expression of other hexokinases including HK I and HK III were studied in the mitochondrial and cytosolic fractions by Western blotting. Results show that expression of HK I and III is not appreciably affected by Hsp90 blockade with 17-DMAG ( $100 \mathrm{nM}, 72 \mathrm{~h}$ or Tom 40 siRNA to a degree that is likely linked to decreased mitochondrial activity (Figure $4 \mathrm{a}$ and $4 \mathrm{~b}$ ). Interestingly, a slight to moderate increase in Hsp90 and Tom 40 protein expression was observed in both the cytosolic and mitochondrial fractions when cells were treated with 17-DMAG. However, this effect was not observed in cells with Tom40 knockdown. Nevertheless, VDAC expression in the mitochondrial fraction was substantially decreased upon inhibition of either Hsp90 or Tom40 (Figure $4 \mathrm{a}$ and $4 \mathrm{~b}$ ). This suggests that both treatments disturb Tom40 localization and activity and, in consequence, cause a change in intracellular distribution of VDACs and HK II. Interestingly, however, in the case of siRNA against Tom40 this occurred through decreased expression of Tom40, whilst Hsp90 inhibition resulted in an increase in mitochondrial Hsp90 and Tom 40. Hence, these effects were subsequently evaluated further.

A

TMK-1

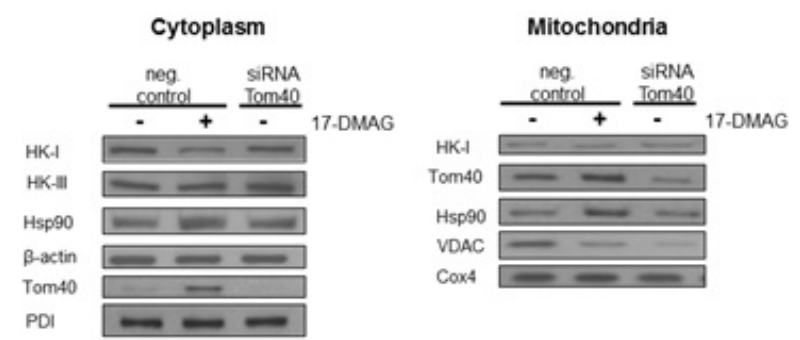

B

MiaPaCa-2

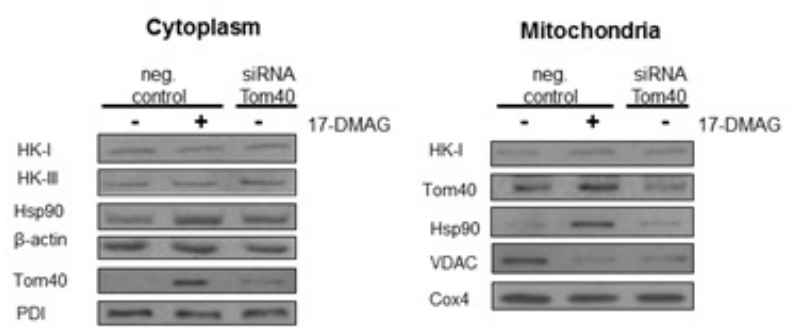

Figure 4: Cytoplasmic and mitochondrial protein expression upon Hsp90 inhibition and Tom40 blockade.

Assigned proteins were determined by Western blotting of TMK-1 (a) and MiaPaCa-2 (b) cells. No significant differences were observed in $\mathrm{HK}$ I and III expression levels in the cytoplasmic fraction. Nevertheless, Hsp90 and Tom 40 expression were increased in cytoplasmic and mitochondrial fractions after treatment with 17-DMAG (100 nM, $72 \mathrm{~h}$ ) in both cell lines. Mitochondrial VDAC expression was reduced in the presence of both 17-DMAG and Tom40 siRNA.

\section{Co-immunoprecipitation of Tom40 with Hsp90}

To explain the increased abundance of Tom 40 and Hsp90 protein in the mitochondrial fraction after 17-DMAG treatment, co-immunoprecipitation of mitochondrial Tom40 with Hsp90 was performed. There was a clear increase in the abundance of Hsp90 precipitated with Tom40 protein (Figure 5). This supports the idea that the increase in mitochondrial localisation of Hsp90 and Tom 40 is due to a greater number of un-dissociated Hsp90/Tom 40 complexes. This finding is a critical observation, because proteins require separation from Hsp90 to acquire proper folding.

\begin{tabular}{|c|c|c|c|c|c|}
\hline \multirow{3}{*}{ TMK-1 } & \multirow[b]{3}{*}{ Hsp90 } & \multicolumn{4}{|c|}{$\begin{array}{l}\text { co-IP Tom40 } \\
\text { mitochondrial protein }\end{array}$} \\
\hline & & ctl. WB & mock & ctl. & 17-DMAG \\
\hline & & - & & +6 & (4) \\
\hline & Tom40 & +2 & + & & \\
\hline
\end{tabular}

Figure 5: Reduced dissociation of Hsp90/Tom40 in mitochondria after Hsp90 inhibition with 17-DMAG.

Mitochondrial protein was extracted from the cells kept under control conditions or treated with 17-DMAG (100 nM, $72 \mathrm{~h}$ ). The first line (ctl WB) represents western blot performed on the non-co-immunoprecipitated protein. A control without antibody addition in the co-IP incubation mixture was performed (mock). There was an increase in Hsp90-Tom40 complexes upon Hsp90 inhibition, compared to control conditions. Results are shown for TMK-1; similar results were obtained for MiaPaCa2. 


\section{Metabolic analysis}

HPLC-ESI-MS/MS analysis of intracellular succinate extracted from cells grown under assigned conditions was performed. Inhibition of Hsp90 with 17-DMAG (100 nM, 72 h) and siRNA-mediated Tom 40 knockdown, respectively, led to a significant decrease in mitochondrial activity, as determined by the rate of synthesis of succinate from fully ${ }^{13} \mathrm{C}$-labelled glucose (Figure 6a). Analysis of media regarding glycolysis products of cells treated with 17-DMAG showed a small, but significant increase in the release of lactate into the culture media (Figure $6 \mathrm{~b}$ ). To further check for the changes in the glycolytic activity of the studied cells measurements of the release/synthesis of serine and glycine were performed. A significant increase in excretion of ${ }^{13} \mathrm{C}$-labelled serine was observed in both cell lines as well as an increase in release of ${ }^{13} \mathrm{C}$-labelled glycine in TMK-1 cells (Figure 6C). Serine and glycine are present in the culture medium and can be taken up by the cells. To illustrate extracellular changes in these metabolites we also plotted peak areas for ${ }^{12} \mathrm{C}$ (uptake, shown as remaining substrate as compared to the level in blank media; Figure 6d). Cells treated with 17-DMAG (100 nM, 72 h) show less uptake of serine from the media. Together, these results suggest a metabolic shift towards glycolysis (as shown by increase in lactate and serine for both cell lines and glycine in the case of TMK-1) in the presence of decreased mitochondrial activity upon Hsp90 inhibition with 17-DMAG.

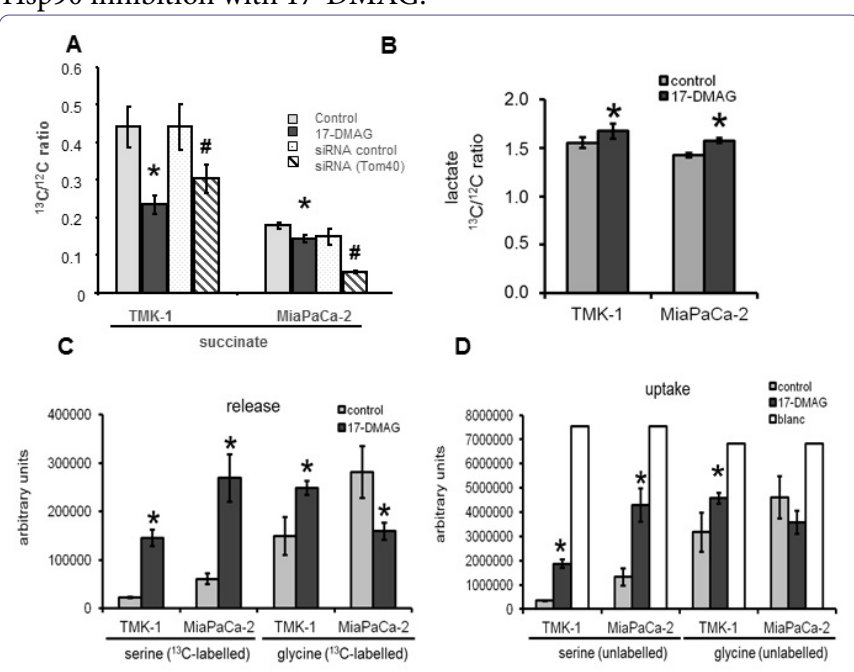

Figure 6: Metabolomics

Metabolomic analysis of cell pellets (a) and media (b-d). $4 \mathrm{mM}$ [U-13C] glucose was added $24 \mathrm{~h}$ after the onset of treatment, which was carried out for a total of $72 \mathrm{~h}$. The ratio of $13 \mathrm{C}$-labelled to unlabeled intracellular succinate is presented for TMK-1 and MiaPaCa-2 a) cell lines A significant reduction of intracellular succinate synthesis was found with Hsp90 inhibitor present $\left({ }^{*} \mathrm{P}<0.05\right)$ and Tom40 blockade $(\# \mathrm{P}<0.05)$. b) lon ratios for lactate in media indicate a significant increase in lactate secretion after 17-DMAG treatment. c) Release of 13C-labelled serine was significantly enhanced in both cell lines ( $\left.{ }^{\star} \mathrm{P}<0.05\right)$. In TMK-1, an increase in glycine release was also observed. d) Cellular uptake of serine was decreased significantly upon Hsp90 inhibition, whereas only insignificant changes were found concerning glycine uptake. White histobars represent glycine and serine level in media. ( $n=3$ per group; $\left.{ }^{*} \mathrm{P}<0.05\right)$

\section{In vivo experiments}

The experiments were substantiated by in vivo experiments. A subcutaneous syngenic mouse model with murine PancO2 pancreatic cancer cells was used. Mice were treated with 17-DMAG (25 mg/kg loading-dose, $10 \mathrm{mg} / \mathrm{kg} / \mathrm{d}$ as a maintenance dose).
Hexokinase activity and protein expression in cytosolic and mitochondrial fractions was assessed in tumor specimens extracted from control and 17-DMAG-treated animals. Results show that while total cytosolic hexokinase activity did not differ between the two groups ( $\mathrm{n}=3$ each), mitochondrial hexokinase activity was reduced significantly in tumors of 17-DMAG-treated mice (Figure 7a). Moreover, determination of the subcellular protein expression using Western blotting confirmed our in vitro results; mitochondrial VDAC expression was decreased, while Tom40 and Hsp90 levels were increased (Figure $7 \mathrm{~b}$ ).

A

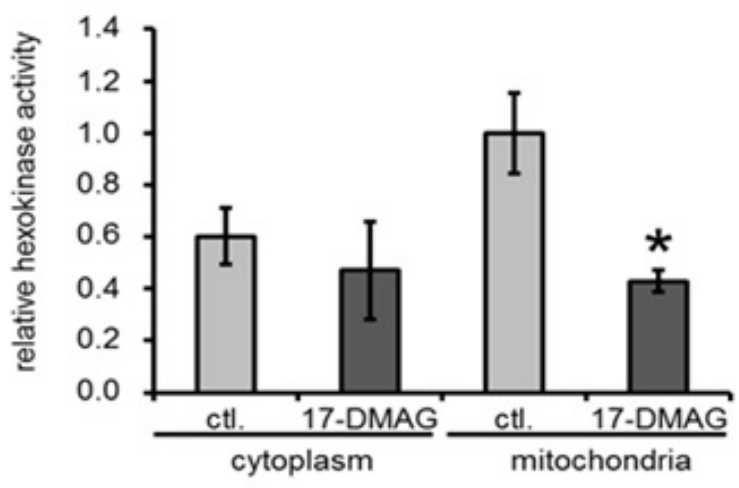

B

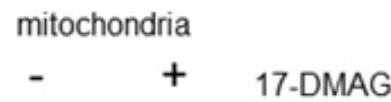

VDAC

Tom40

Hsp90

Cox4

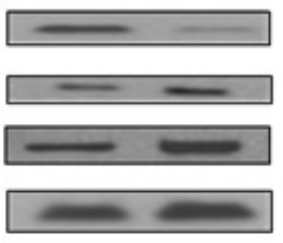

Figure 7: In vivo experiments.

a) Mitochondrial and cytoplasmic fractions extracted from mouse tumors were analyzed for HK II activity. A significant inhibition of mitochondrial HK II activity was observed upon treatment with $17-D M A G\left({ }^{*} P<0.05 ; n=8\right)$. b) The mitochondrial tissues. Western blotting revealed a decrease in VDACs and an increase in Tom40 and Hsp90 expression.

\section{Discussion}

Hsp90 plays a crucial role in oncogenesis by sustaining proper folding and trafficking of oncoproteins [2]. Therefore, Hsp90 inhibitors are studied intensively as potential anti-cancer drugs [21]. Here, we have focused on the effects of Hsp90 interactions with the mitochondrial protein Tom40. While our study does not exclude that other proteins are also involved in metabolic control through the Hsp90 inhibition, similar effects of chaperone inhibition and Tom 40 knock-down do suggest Tom40 interactions with Hsp90 are a major cause of the observed metabolic alterations such as mitochondrial disruption and increase in glycolytic activity. Interestingly, the role of Hsp90 in cancer cell metabolism, in particular in glycolysis and mitochondrial respiration, has not been examined in detail. In the present study, we reveal a novel mechanism of Hsp90 action on cancer cell metabolism. 
Our experiments show, that blocking Hsp90 in cancer cells results in a shift of cellular metabolism towards glycolysis, reduced Krebs cycle activity, and a change in subcellular localization of metabolism-related proteins. In the absence of changes in overall protein expression [11], we show that sub-cellular localization of HK II is substantially affected by Hsp90 blockade. This effect was the result of an altered interaction of Hsp90 with its client protein Tom 40 $[12,13]$, which is a part of the greater TOM complex [22]. Inhibition of Hsp90 resulted in higher Tom40 and Hsp90 abundance in the mitochondria. However, co-immunoprecipitation experiments showed that the observed increase was due to the presence of undissociated Hsp90-Tom40 complexes in the organelles. 17-DMAG is known to block the ATP site of Hsp90, which is needed for proper dissociation of the client protein from the chaperone [23]. Apparently, these complexes still target mitochondria but their inability to acquire proper TOM complex function triggers overexpression of Tom 40 in the mitochondria and cytoplasm. This finding is consistent with the observation that ATP depletion results in Tom 40 being arrested in large complexes consisting of Tom40, TOM machinery and cytosolic chaperones [12]. Therefore, our study shows, that while these Tom40-Hsp90 complexes still traffic to mitochondria, they do not dissociate in the presence of 17-DMAG. Interestingly, the use of novobiocin which is known to degrade Hsp90 client protein [24] reduces incorporation of Tom 40 into the mitochondrial membrane [13]. A similar reduction was also observed when competitive Hsp90 binding was applied [12]. In all the cases, including the present research, Hsp90 disruption resulted in a reduced or faulty incorporation of Tom 40 into the mitochondrial membrane. It can therefore be reasonably deduced that Hsp90 takes part in trafficking of Tom 40 to the mitochondrial membrane. Interestingly, a significant role of the mitochondrial Hsp90 in keeping energy production in cancer cells has been observed recently $[25,26]$. In can be, therefore, assumed that blocking Hsp90 activity results in mitochondrial disruption and metabolic changes, however, the exact mechanism depends on the drug used and the part of Hsp90 affected.

Most mitochondrial proteins are coded for in the nucleus and are subsequently transferred through the mitochondrial membranes. Therefore, TOM disruption is expected to affect the abundance of several crucial molecules in this organelle, e.g. ion channels and transporters [27] that are responsible for proper exchange and communication between the mitochondria and cytoplasm. In our study we have focused on one of the major glycolytic enzymes, HK II. To acquire proper activity this enzyme needs to be attached to VDAC (believed to supply ATP for HK II; [28]), which is one of the most abundant and crucial mitochondrial communication channels [29]. We found that long-term inhibition of Hsp90 results in decreased VDAC expression in mitochondria and, in consequence, reduced abundance of HK II binding to the mitochondrial channels. We conclude, that Hsp90 inhibition affects mitochondrial import of VDACs and, thus, binding of HK II to the outer mitochondrial membrane.

We also studied the consequences of Hsp90 inhibition-mediated changes to Tom40, HK II and VDAC on the Krebs cycle and glycolysis. Krebs cycle activity was determined as the ratio of incorporation of ${ }^{13} \mathrm{C}$ from ${ }^{13} \mathrm{C}$ labelled glucose into succinate, and was found to be decreased. This effect was expected because of the inability of mitochondria to properly exchange metabolites with the cytoplasm, resulting in Krebs cycle inhibition. In our research we have focused on VDACs abundance in the mitochondria in the presence of Hsp90 blocker. However, since TOM complex is involved in the import of most mitochondrial proteins, decreased mitochondrial activity is more likely related to the overall inhibited incorporation of several mitochondrial proteins including metabolic transporters such as pyruvate [30] or citrate [31]. Disruption in the abundance of mitochondrial channels and transporters could also alter mitochondrial membrane potential, additionally affecting Krebs cycle activity; this potential aspect will require further investigation.

Glycolytic activity was assessed by measuring the ratio of incorporation of ${ }^{13} \mathrm{C}$ from ${ }^{13} \mathrm{C}$ labelled glucose into extracellular lactate, serine and glycine. Despite the fact that HK II binding to VDACs was decreased, overall glycolytic activity was increased. There was an accumulation of lactate, serine and, in the case of the gastric cancer cell line, glycine in the cells and supernatant. The increase in lactate might be explained by the reduced utilization of pyruvate in the Krebs cycle. As a consequence, it is rather converted to lactate. Interestingly, glycolytic activity shifted mainly towards serine/glycine synthesis in both cell lines. Serine is produced by phosphoglycerate dehydrogenase from 3-phosphoglycerate, one of the intermediates of the glycolytic pathway [32]. This shift could be explained by excess serine serving as a substrate to sustain metabolic equilibrium in the cells. Importantly, serine and glycine are precursors of many biosynthetic pathways leading to the production of amino acids, purines and pyrimidines. Additionally, during the conversion of 3-phosphoglycerate to serine, glutamate is metabolized into $\boldsymbol{\alpha}$-ketoglutarate [32]; $\boldsymbol{\alpha}$-ketoglutarate, in turn, is a precursor of other metabolites (e.g. citrate) necessary for fatty acid synthesis. Moreover, serine synthesis is accompanied by NADH production needed to maintain red/ox balance. Therefore, enhancement of this serine-producing pathway via Hsp90 inhibition could partially compensate for the reduced abundance of cytosolic metabolites normally supplied by mitochondria. In fact, increased synthesis of serine and glycine derived from glycolysis has been associated with some types of cancer [33,34]. The slight differences in regulation of the serine/glycine ratio observed in the two cancer cell lines studied is likely due to the cells originating from different tissues (pancreas versus stomach).

Previous research has shown that targeting Hsp90 reduces tumor growth in vivo $[10,16]$; in vitro studies suggest this effect may be related to increased cell death [35]. However, we speculate from our results that although the overall cell number and tumor growth may be reduced under Hsp90 inhibition, the surviving cells will likely retain metabolic characteristics that potentially correlate with a high metastatic potential, such as increased glycolysis and serine synthesis. This finding raises concerns about Hsp90 inhibitory therapy that needs to be further researched.

\section{Conclusions}

To conclude, the present study shows that Hsp90 inhibition reduces mitochondrial respiration and increases glycolysis in the studied cancer cells, shifting metabolism mainly towards serine/glycine synthesis. These changes occur due to the disrupted interactions between $\mathrm{Hsp} 90$ and Tom40 indicating that Hsp90 supports mitochondrial biogenesis by assuring assembly of TOM. The overall shift towards glycolysis (a phenomenon associated with metastatic behavior of cancer cells) due to disrupted trafficking of mitochondrial proteins should be considered when applying Hsp90 inhibitors in clinics. 


\section{Acknowledgment}

These studies were supported by the German Research Council (DFG/KFO262/La1988/3-1 and LA1988/2-1) to SAL and (DFG/KFO262/De835/2-1) to KD.

\section{References}

1. Picard D (2002) Heat-shock protein 90, a chaperone for folding and regulation. Cell Mol Life Sci 59: 1640-1648.

2. Trepel J, Mollapour M, Giaccone G, Neckers L (2010) Targeting the dynamic HSP90 complex in cancer. Nat Rev Cancer 10: 537-549.

3. Jin S, DiPaola RS, Mathew R, White E (2007) Metabolic catastrophe as a means to cancer cell death. J Cell Sci 120: 379-383.

4. Warburg $\mathrm{O}(1956)$ On respiratory impairment in cancer cells. Science 124: 269-270.

5. Qiu MZ, Han B, Luo HY, Zhou ZW, Wang ZQ, et al. (2011) Expressions of hypoxia-inducible factor- $1 \alpha$ and hexokinase-II in gastric adenocarcinoma: the impact on prognosis and correlation to clinicopathologic features. Tumour Biol 32: 159-166.

6. Natsuizaka M, Ozasa M, Darmanin S, Miyamoto M, Kondo S, et al. (2007) Synergistic up-regulation of Hexokinase-2, glucose transporters and angiogenic factors in pancreatic cancer cells by glucose deprivation and hypoxia. Exp Cell Res 313: 3337-3348.

7. Peng Q, Zhou Q, Zhou J, Zhong D, Pan F, et al. (2008) Stable RNA interference of hexokinase II gene inhibits human colon cancer LoVo cell growth in vitro and in vivo. Cancer BiolTher 7: 1128-1135.

8. Mathupala SP, Ko YH, Pedersen PL (2010) The pivotal roles of mitochondria in cancer: Warburg and beyond and encouraging prospects for effective therapies. Biochim Biophys Acta 1797: 1225-1230.

9. Shulga N, Wilson-Smith R, Pastorino JG (2010) Sirtuin-3 deacetylation of cyclophilin $\mathrm{D}$ induces dissociation of hexokinase II from the mitochondria. J Cell Sci 123: 894-902.

10. Smith-Jones PM, Solit D, Afroze F, Rosen N, Larson SM (2006) Early tumor response to Hsp90 therapy using HER2 PET: comparison with 18F-FDG PET. J Nucl Med 47: 793-796.

11. Yao JQ, Liu QH, Chen X, Yang Q, Xu ZY, et al. (2010) Hsp90 inhibitor 17-allylamino-17-demethoxygeldanamycin inhibits the proliferation of ARPE-19 cells. J Biomed Sci 17: 30.

12. Humphries AD, Streimann IC, Stojanovski D, Johnston AJ, Yano M, et al. (2005) Dissection of the mitochondrial import and assembly pathway for human Tom40. J Biol Chem 280: 11535-11543.

13. Joseph AM, Hood DA (2012) Plasticity of TOM complex assembly in skeletal muscle mitochondria in response to chronic contractile activity. Mitochondrion 12: $305-312$.

14. Miccoli L, Oudard S, Sureau F, Poirson F, Dutrillaux B, et al. (1996) Intracellular $\mathrm{pH}$ governs the subcellular distribution of hexokinase in a glioma cell line. Biochem J 313 : 957-962.

15. Lang SA, Gaumann A, Koehl GE, Seidel U, Bataille F, et al. (2007) Mammalian target of rapamycin is activated in human gastric cancer and serves as a target for therapy in an experimental model. Int J Cancer 120: 1803-1810.

16. Lang SA, Moser C, Gaumann A, Klein D, Glockzin G, et al. (2007) Targeting heat shock protein 90 in pancreatic cancer impairs insulin-like growth factor-I receptor signaling, disrupts an interleukin-6/signal-transducer and activator of transcription 3/hypoxia-inducible factor-1alpha autocrine loop, and reduces orthotopic tumor growth. Clin Cancer Res 13: 6459-6468.

17. Mazurek MP, Prasad PD, Gopal E, Fraser SP, Bolt L, et al. (2010) Molecular origin of plasma membrane citrate transporter in human prostate epithelial cells. EMBO Rep 11: 431-437.
18. Dettmer K, Nürnberger N, Kaspar H, Gruber MA, Almstetter MF, et al. (2011) Metabolite extraction from adherently growing mammalian cells for metabolomics studies: optimization of harvesting and extraction protocols. Anal Bioanal Chem 399: 1127-1139.

19. van der Goot AT, Zhu W, Vázquez-Manrique RP, Seinstra RI, Dettmer K, et al. (2012) Delaying aging and the aging-associated decline in protein homeostasis by inhibition of tryptophan degradation. Proc Natl Acad Sci USA 109: 14912-14917.

20. Wachsmuth CJ, Almstetter MF, Waldhier MC, Gruber MA, Nürnberger N, et al. (2011) Performance evaluation of gas chromatography-atmospheric pressure chemical ionization-time-of-flight mass spectrometry for metabolic fingerprinting and profiling. Anal Chem 83: 7514-7522.

21. Mahalingam D, Swords R, Carew JS, Nawrocki ST, Bhalla K, et al. (2009) Targeting HSP90 for cancer therapy. Br J Cancer 100: 1523-1529.

22. Rapaport D (2005) How does the TOM complex mediate insertion of precursor proteins into the mitochondrial outer membrane? J Cell Biol 171: 419-423.

23. Onuoha SC, Mukund SR, Coulstock ET, Sengerovà B, Shaw J, et al. (2007) Mechanistic studies on $\mathrm{Hsp} 90$ inhibition by ansamycin derivatives. J Mol Biol 372: $287-297$.

24. Donnelly A, Blagg BS (2008) Novobiocin and additional inhibitors of the Hsp90 C-terminal nucleotide-binding pocket. Curr Med Chem 15: 2702-2717.

25. Chae YC, Caino MC, Lisanti S, Ghosh JC, Dohi T, et al. (2012) Control of tumor bioenergetics and survival stress signaling by mitochondrial HSP90s. Cancer Cell 22: 331-344

26. Chae YC, Angelin A, Lisanti S, Kossenkov AV, Speicher KD, et al. (2013) Landscape of the mitochondrial Hsp90 metabolome in tumours. Nat Commun 4: 2139.

27. Rapaport D, Neupert W (1999) Biogenesis of Tom40, core component of the TOM complex of mitochondria. J Cell Biol 146: 321-331.

28. Gottlob K, Majewski N, Kennedy S, Kandel E, Robey RB, et al. (2001) Inhibition of early apoptotic events by Akt/PKB is dependent on the first committed step of glycolysis and mitochondrial hexokinase. Genes Dev 15: 1406-1418.

29. Shoshan-Barmatz V, Ben-Hail D (2012) VDAC, a multi-functional mitochondrial protein as a pharmacological target. Mitochondrion 12: 24-34.

30. Bricker DK, Taylor EB, Schell JC, Orsak T, Boutron A, et al. (2012) A mitochondrial pyruvate carrier required for pyruvate uptake in yeast, Drosophila, and humans. Science 337: 96-100.

31. Zara V, Ferramosca A, Papatheodorou P, Palmieri F, Rassow J (2005) Import of rat mitochondrial Citrate Carrier $(\mathrm{CIC})$ at increasing salt concentrations promotes presequence binding to import receptor Tom 20 and inhibits membrane translocation. J Cell Sci 118: 3985-3995.

32. DeBerardinis RJ (2011) Serine metabolism: some tumors take the road less traveled. Cell Metab 14: 285-286.

33. Jain M, Nilsson R, Sharma S, Madhusudhan N, Kitami T, et al. (2012) Metabolite profiling identifies a key role for glycine in rapid cancer cell proliferation. Science 336: 1040-1044.

34. Locasale JW, Grassian AR, Melman T, Lyssiotis CA, Mattaini KR, et al. (2011) Phosphoglycerate dehydrogenase diverts glycolytic flux and contributes to oncogenesis. Nat Genet 43: 869-874.

35. Karkoulis PK, Stravopodis DJ, Margaritis LH, Voutsinas GE (2010) 17-Allylamino-17-demethoxygeldanamycin induces downregulation of critical Hsp90 protein clients and results in cell cycle arrest and apoptosis of human urinary bladder cancer cells. BMC Cancer 10: 481 